\title{
5-Fluorouracil induces an acute reduction in neurogenesis and persistent neuroinflammation in a mouse model of the neuropsychological complications of chemotherapy
}

Courtney Brooke Subramaniam ( $\square$ courtney.subramaniam@adelaide.edu.au )

The University of Adelaide School of Medical Sciences: The University of Adelaide Adelaide Medical School https://orcid.org/0000-0002-9549-5346

Hannah R. Wardill

The University of Adelaide

Maya R. Davies

The University of Adelaide

\section{Vivien Heng}

The University of Adelaide

\section{Marc A. Gladman}

The University of Adelaide

Joanne M. Bowen

The University of Adelaide

\section{Research Article}

Keywords: chemotherapy, neuropsychological deficits, neuroinflammation, neurogenesis, supportive oncology

Posted Date: February 11th, 2022

DOI: https://doi.org/10.21203/rs.3.rs-1337266/v1

License: (c) (i) This work is licensed under a Creative Commons Attribution 4.0 International License. Read Full License 


\section{Abstract}

The neuropsychological symptoms of chemotherapy treatment remain a major challenge with their prevention hampered by insufficient understanding of pathophysiology. While long term neuroimmune changes have been identified as a hallmark feature shared by neurological symptoms, the exact timeline of mechanistic events preceding and driving neuroinflammation remain unclear. We therefore aimed to longitudinally characterise the neuroimmunological changes following systemic 5-fluorouracil (5-FU) to gain insight into the timeline of events preceding the well-documented chronic neuroinflammation seen following chemotherapy. Eighteen female $\mathrm{C} 57 \mathrm{BI} / 6$ mice received a single intraperitoneal dose of 5-FU and groups were killed at days 1 and 2 (acute timepoint), days 4 and 8 (subacute timepoint) as well as days 16 and 32 (chronic timepoint). A further six mice were used to control for the effect of ageing and stress, with tissues collected on day 1 and day 32. Levels of neurogenesis were determined through immunofluorescent staining of doublecortin (DCX). The activation of microglia and astrocytes were assessed using immunofluorescence staining of ionised calcium binding adaptor molecular-1 (Iba1) and glial fibrillary acidic protein (GFAP) respectively. 5-FU treatment caused significant decreases to DCX staining $(p=0.0030)$ as well as increases in microglial activation in the prefrontal cortex $(p=0.0256)$, CA3 region $(p=0.0283)$ and dentate gyrus $(p=0.0052)$ of the hippocampus at acute timepoints. Whereas astrocytic reactivity was observed across multiple brain regions at subacute and chronic timepoints. This study has identified acute objective neuroinflammatory changes suggesting that the role of early intervention should be explored to prevent the development of neuropsychological deficits following chemotherapy.

\section{Introduction}

Even in the era of novel therapies, chemotherapy remains the cornerstone of successful anti-cancer treatment for the majority of patients. Despite improvements in clinical efficacy, its use remains associated with severe side effects due to non-selective cytotoxicity that impacts nearly all body systems, including the central nervous system (CNS) [1]. The neuropsychological complications of chemotherapy encompass a constellation of neurological deficits that include typical cognitive impairments affecting memory, executive function, processing speeds and learning [2-4], in addition to psychological elements such as depression/anxiety, fear of recurrence and personality changes $[5,6]$. These complications are particularly burdensome due to the chronicity of symptoms which persist long after treatment cessation, ultimately limiting employment prospects and severely impacting quality of life [7]. With cancer management increasingly being focussed on survivorship, there is now a heightened attention on minimising late treatment effects, with neuropsychological symptoms consistently identified as an unmet area of need by cancer survivors [8].

Increasing evidence of neuropsychological symptom clusters has been observed in individuals treated with chemotherapy, suggestive of shared aetiological mechanisms $[9,10]$. Despite this, studies have failed to investigate the timeline of pathological events initiated by chemotherapy, which may be common to the global development of neuropsychological symptom clusters. Neuroimmune changes 
have been identified as a hallmark feature shared by these symptoms [11], suggesting that chemotherapy-induced neuroinflammation plays an important role in the pathophysiology of numerous neurological symptoms. Notably, chronic neuroinflammation has been strongly implicated in other clinical settings such as neurodegeneration and depression as well as fear and anxiety-based disorders [12-14]. While chronic neuroinflammation induced by chemotherapy treatment presents as a convincing mechanistic candidate, the exact timeline of potential mechanistic events occurring in the acute, subacute and/or chronic setting following chemotherapy, and their relationship with chronic neuroinflammation, remain unclear.

Microglia and astrocytes, key innate immune cells within the CNS [15], play a role in chemotherapyinduced neuroinflammation, with their activation seen following the systemic administration of chemotherapy drugs [16]. However, this role has largely been established through cross-sectional preclinical studies which often focus on one outcome measure, analysed at one timepoint after chemotherapy. As such, the relationship between microglia and astrocytes following chemotherapy, including whether the chronic neuroinflammatory response is driven by one or both, remains to be defined. Furthermore, the cross-sectional design employed by preclinical studies has prevented investigation into the events preceding glial cell activation. This is particularly important to understand as gliosis most commonly occurs in response to a stress stimulus. Consequently, there is likely to be a triggering event responsible for glial activation followed by subsequent neuroinflammation.

Activation of glial cells can occur secondary to direct cytotoxic injury [6, 17], as seen in other organs systems and cell populations exposed to chemotherapy [18]. However, this mechanism remains controversial, as most chemotherapeutic drugs are not capable of crossing the highly restrictive blood brain barrier (BBB). Further, direct cytotoxic effects are difficult to rationalise given the propensity of chemotherapeutic drugs to induce cytotoxic injury to rapidly dividing cell populations, and the relatively low turnover rate of most CNS cells. While these strongly held beliefs have challenged our understanding of the aetiology of neuropsychological symptoms, it is important to consider that: i) some chemotherapeutic drugs, particularly those with low molecular weights (e.g. 5-fluorouracil), are capable of crossing the BBB, ii) BBB hyperpermeability has been reported after chemotherapy, increasing paracellular transport [19], and iii) there are subpopulations of cells in the brain that are in fact highly proliferative.

Preclinical studies have shown certain chemotherapy drugs significantly reduce the number of actively proliferating neurons and immature neurons within the dentate gyrus of the hippocampus, a key site of adult neurogenesis [20-22], providing critical insight into the damaging effect of chemotherapy on proliferating neurons. Mechanistically, this direct cytotoxic injury may be sufficient to induce oxidative stress and resulting inflammation, which, with repeated doses of chemotherapy, would create sustained and chronic neuroinflammation. However, since analyses were only performed in the chronic setting following treatment, it is difficult to solely attribute causality to direct cytotoxicity when the prolonged stress and systemic inflammation commonly associated with chemotherapy may also contribute to impaired neurogenesis over such a sustained period of time [23-25]. We therefore aimed to longitudinally 
characterise the neuroimmunological changes of 5-fluorouracil (5-FU) treatment in order to gain insight into the timeline of events preceding the well-documented chronic neuroinflammation seen following chemotherapy. Specifically, we hypothesised that acute changes to neurogenesis would precede glial cell activation following administration of 5-FU.

\section{Star Methods}

This study was reported using the STAR methods for structured, transparent and accessible reporting as well as the ARRIVE guidelines for the accurate and reproducible reporting of animal research.

\subsection{Experimental Model Details}

\subsubsection{Ethical statement and animal husbandry}

The study was approved by the Animal Ethics Committee of the South Australian Health and Medical Research Institute (SAHMRI, \#SAM20-034) and complied with the National Health and Research Council (Australia) Code of Practice for Animal Care in Research and Training (2014). All experiments were conducted on female C57BI/6 mice, obtained from the SAHMRI Bioresources Facility, aged 13 weeks at arrival and 14 weeks following 7 days of habituation prior to study commencement. Mice were grouphoused (maximum of 5 animals/cage) in individually ventilated cages, under specific pathogen-free conditions at the SAHMRI Bioresources Facility (Adelaide, SA, Australia). They were housed under a 12hour light-dark cycle (lights on at 7:00 a.m.), at a constant temperature of $20-23^{\circ} \mathrm{C}$ and humidity maintained between $45-75 \%$, with ad libitum access to water and a standard chow diet (Teklad Global Diet \#2918, Envigo). Sawdust and crinkle bedding, along with a toilet roll for enrichment, were provided in all cages. To encourage normal feeding behaviour following treatment, all mice were provided with soaked chow (standard chow softened with water to ease mastication) in a petri dish, on the cage floor. Mice were weighed daily and in the case of weight loss greater than $10 \%$ (an expected treatment-related toxicity), they were provided with peanut butter (Bega Peanut Butter, Smooth) and DietGel 31M (Clear $\left.\mathrm{H}_{2} \mathrm{O} ; 72-08-5022\right)$ in a petri dish, on the cage floor as well as a heat mat to prevent hypothermia.

\subsubsection{Study design}

Mice were treated with a single $400 \mathrm{mg} / \mathrm{kg}$ dose of 5-FU (Sigma Aldrich; F6627-10G), reconstituted at 10 $\mathrm{mg} / \mathrm{mL}$ in sterile saline, via intraperitoneal injection (day 0 ). Following treatment, mice were randomly allocated into 6 experimental groups ( $\mathrm{N}=3$ /group), with tissues collected from each group at days 1 and 2 (acute timepoints), 4 and 8 (subacute timepoints) as well as 16 and 32 (chronic timepoints). A further 6 mice, who did not receive 5-FU, were used to control for the effect of ageing and stress due to repeated handling on outcome measures, with tissues being collected from $\mathrm{N}=3$ mice on day 1 and $\mathrm{N}=3$ mice on day 32 of the study (see supplementary Fig. 1).

\subsubsection{Tissue collection}


All animals were anesthetised via inhalation isoflurane ( $2 \%$ in $1 \mathrm{~L} / \mathrm{min}$; Provet) and administered with 0.1 $\mathrm{mL}$ of sodium pentobarbitone $(325 \mathrm{~g} / \mathrm{mL}$, Lyppard) intraperitoneally before transcardial perfusion with 20 $\mathrm{mL}$ of sterile saline (Vital Medical Supplies). The brains were dissected, with the left hemisphere snap frozen in liquid nitrogen and stored at $-80^{\circ} \mathrm{C}$ and the right hemisphere post-fixed in $10 \%$ formalin (Southern Cross Science) for 24 hours prior to processing. The primary outcome measures for this study comprised of validated markers of neuroinflammation, neurogenesis and apoptosis. All analyses were performed blinded.

\subsection{Method Detail}

\subsubsection{RNA extraction and real time polymerase chain reaction}

RNA was extracted from the frontal cortex of the left hemisphere using the NuceloSpin RNA mini kit for RNA purification (Macherey-Nagel; 740984.250) as per the manufacturer's instructions. Briefly, frozen samples were homogenised, at room temperature, in 350 $\mathrm{L}$ of lysis buffer (Macherey-Nagel; 740984.250) using the Qiagen TissueLyser LT (Qiagen) for 5 minutes at $50 \mathrm{~Hz}$. Following a series of filtration, DNAdigestion and washing steps, highly purified RNA was eluted in RNase-free $\mathrm{H}_{2} \mathrm{O}$ and stored at $-80^{\circ} \mathrm{C}$. Using a Nanodrop 100 spectrophotometer (Thermo Scientific), RNA yield and purity was determined. $1 \mu \mathrm{g}$ of RNA from each sample was then reverse transcribed, as per the manufacturers' instructions, using the iScript ${ }^{\text {TM }}$ CDNA Synthesis Kit (BioRad; 1706891). RT-PCR was then performed using the Rotor-Gene 3000 (Corbett Research) to determine the level of expression of multiple genes of interest (Table 1). $10 \mu \mathrm{L}$ amplification mixes comprised of $1 \mu \mathrm{L}$ of cDNA (prediluted to $100 \mathrm{ng} / \mu \mathrm{L}$ ), $5 \mu \mathrm{L}$ of QuantiTect SYBR green assay reagents (Qiagen; 204143), $3 \mu \mathrm{L}$ of RNase-free $\mathrm{H}_{2} \mathrm{O}$ and $0.5 \mu \mathrm{L}$ of both the forward and reverse primers for the gene of interest (prediluted to $50 \mathrm{pmol} / \mu \mathrm{L}$ ). Primer sequences are detailed in Table 1 . Thermal cycling conditions included an initial denaturing step at $95^{\circ} \mathrm{C}$ for 10 minutes, followed by 40 cycles of denaturation at $95^{\circ} \mathrm{C}$ for 15 seconds and annealing at $60^{\circ} \mathrm{C}$ for 40 seconds. All samples were run in triplicate and cycle threshold (ct) values were calculated by the RotorGene 6 analysis software. The expression level for each gene of interest was calculated relative to the housekeeper gene $\beta$-actin using the $\Delta$ ct method, a variation of the Livak method [26]. 
Table 1

RT-PCR primer specifications

\begin{tabular}{|c|c|c|}
\hline Target & Sequence $5^{\prime}-3^{\prime}$ & $\operatorname{Tm}\left({ }^{\circ} \mathrm{C}\right)$ \\
\hline \multirow[t]{2}{*}{ BCL2 } & F: TAC CGT CGT GAC TTC GCA GAG & \multirow[t]{2}{*}{$67.6 ; 70.2$} \\
\hline & R: GGC AGG CTG AGC AGG GTC TT & \\
\hline \multirow[t]{2}{*}{ BDNF } & F: TGC AGG GGC ATA GAC AAA AGG & \multirow[t]{2}{*}{$68.1 ; 68.3$} \\
\hline & R: CTT ATG AAT CGC CAG CCA ATT CTC & \\
\hline \multirow[t]{2}{*}{ TIMP-1 } & F: GGC ATC TGG CAT CCT CTT GT & \multirow[t]{2}{*}{$66.5 ; 65.6$} \\
\hline & R: CGC TGG TAT AAG GTG GTC TCG & \\
\hline \multirow[t]{2}{*}{ IL-6R } & F: TGA ATG ATG ACC CCA GGC AC & \multirow[t]{2}{*}{$57.5 ; 57.6$} \\
\hline & R: ACA CCC ATC CGC TCT CTA CT & \\
\hline \multirow[t]{2}{*}{ MMP-2 } & F: CTG ATA ACC TGG ATG CCG TCG & \multirow[t]{2}{*}{$68.7 ; 67.6$} \\
\hline & R: CTG GTG TGC AGC GAT GAA GAT & \\
\hline \multirow[t]{2}{*}{ MMP-9 } & F: CGC TCA TGT ACC CGC TGT AT & \multirow[t]{2}{*}{$64.9 ; 65.9$} \\
\hline & R: CCG TGG GAG GTA TAG TGG GA & \\
\hline \multirow[t]{2}{*}{ B-actin } & F: CTC TTC CAG СCT TCC TTC CT & \multirow[t]{2}{*}{$56.4 ; 57.9$} \\
\hline & R: AGC ACT GTG TTG GCG TAC AG & \\
\hline
\end{tabular}

\subsubsection{Immunofluorescence}

Formalin-fixed tissue was processed and embedded into paraffin wax. Using a rotary microtome (Leica; RM2235) $5 \mu \mathrm{M}$ sagittal sections of the brain were cut and then mounted onto glass slides (Flex Plus Detection System, Dako; \#K8020). Immunofluorescent analysis was performed for doublecortin (DCX), a protein expressed by neuronal precursor cells within the dentate gyrus of the hippocampus and commonly utilised as a marker of neurogenesis, as well as neuroinflammatory markers glial fibrillary acidic protein (GFAP) and ionised calcium-binding adaptor protein-1 (Iba1), indicators of astrocytic reactivity and microglial activation respectively. Staining was performed using an automated system (AutostainerPlus, Dako; \#AS480). Briefly, sections were dewaxed using xylene and rehydrated in graded ethanol $(100 \%, 90 \%$ and $70 \% \mathrm{v} / \mathrm{v})$ before being subjected to heat-mediated antigen retrieval using the antigen retrieval buffers (Table 2) and the Dako PTLINK pre-treatment module (Dako; \#PT101). Antigen retrieval buffers were preheated to $65^{\circ} \mathrm{C}$ before immersion of slides. Buffer temperature was then brought to $97^{\circ} \mathrm{C}$ for 20 minutes and cooled to $65^{\circ} \mathrm{C}$ before removal of the slides. Following antigen retrieval, slides were placed in the Dako AutostainerPlus (Dako; \#AS480) and stained using the manufacturers guidelines and the reagents (Table 2). All slides were counter-stained using the tertiary nuclei stain DAPI (4', 6- 
diamidino-2-phenylindole, Sigma; D8417) at $1 \mu \mathrm{g} / \mathrm{mL}$ and cover slipped in aqueous mounting medium (Fluoroshield, Sigma; F6182). Negative controls were stained without a primary antibody. Slides were digitally scanned (Axio Scan.Z1, ZEISS) and assessed using the Zeiss Zen Blue 3.1 program. DCX staining was assessed in two regions of the dentate gyrus, the apex and granule cell layer, and an average calculated per animal. Iba1 and GFAP staining was assessed in the hippocampus (CA1, CA3 and dentate gyrus), prefrontal cortex, midbrain and hypothalamus. For staining within the CA1 and CA3 region of the hippocampus, an average value was obtained for CA1a and CA1b as well as CA3a and CA3C respectively. All staining was quantified using Fiji software (ImageJ) to determine the percent area of positive staining.

Table 2

Reagents utilised for immunofluorescence staining

\begin{tabular}{|c|c|c|c|c|}
\hline Marker & $\begin{array}{l}\text { Antigen retrieval } \\
\text { buffer }\end{array}$ & $\begin{array}{l}\text { Blocking } \\
\text { reagent }\end{array}$ & $\begin{array}{l}\text { Primary } \\
\text { antibody }\end{array}$ & Secondary antibody \\
\hline DCX & $\begin{array}{l}\text { Citrate buffer, } \mathrm{pH} \\
6.0(1 \% \mathrm{v} / \mathrm{v} \text { citric } \\
\text { acid, } 1 \% \mathrm{v} / \mathrm{v} \\
\text { trisodium citrate, } \\
0.3 \% \mathrm{v} / \mathrm{v} \text { tween } 20 \\
\left.\text { in distilled } \mathrm{H}_{2} \mathrm{O}\right) \text {. }\end{array}$ & $\begin{array}{l}\text { Normal } \\
\text { horse } \\
\text { serum } \\
\text { (10\% v/v, } \\
\text { Sigma; } \\
\text { H0146) in } \\
\text { distilled } \\
\mathrm{H}_{2} \mathrm{O} \text {. }\end{array}$ & $\begin{array}{l}\text { Rabbit-anti- } \\
\text { doublecortin } \\
\text { (Abcam; } \\
\text { ab18723) at } \\
1 / 250 \text { in NHS } \\
\text { (5\% v/v, Sigma; } \\
\text { H0146). }\end{array}$ & $\begin{array}{l}\text { Alexa fluor, donkey-anti-rabbit } 568 \\
\text { (0.8mg/mL, Life Technologies; } \\
\text { A10042) in BSA (1\% v/v, Sigma; } \\
\text { A604), foetal bovine serum (FBS } \\
2 \% \text { v/v, Bovogen Biologicals; SFBS- } \\
\text { F) in 1x PBS. }\end{array}$ \\
\hline lba1 & $\begin{array}{l}\text { EDTA buffer, } \mathrm{pH} \\
9.0(0.121 \% \mathrm{w} / \mathrm{v} \\
\text { Tris, } 0.037 \% \mathrm{w} / \mathrm{v} \\
\text { EDTA, } 0.03 \% \mathrm{v} / \mathrm{v} \\
\text { tween } 20 \mathrm{in} \\
\left.\text { distilled } \mathrm{H}_{2} \mathrm{O}\right)\end{array}$ & $\begin{array}{l}\text { Bovine } \\
\text { serum } \\
\text { albumin } \\
\text { (BSA 5\% } \\
\text { v/v, Sigma; } \\
\text { A604) in } \\
\text { distilled } \\
\mathrm{H}_{2} \mathrm{O} \text {. }\end{array}$ & $\begin{array}{l}\text { Rabbit-anti- } \\
\text { lba1 (FUJIFILM } \\
\text { Wako; 019- } \\
19741) \text { at } \\
1 / 200 \text { in BSA } \\
\text { (1\% V/v, Sigma; } \\
\text { A604) }\end{array}$ & $\begin{array}{l}\text { Alexa fluor, goat-anti-rabbit } 488 \\
(1 / 1000, \text { Invitrogen; A11034) in } \\
\text { BSA (1\% v/v, Sigma; A604), in 1x } \\
\text { PBS. }\end{array}$ \\
\hline GFAP & $\begin{array}{l}\text { Citrate buffer, } \mathrm{pH} \\
6.0(1 \% \mathrm{v} / \mathrm{v} \text { citric } \\
\text { acid, } 1 \% \mathrm{v} / \mathrm{v} \\
\text { trisodium citrate, } \\
0.03 \% \mathrm{v} / \mathrm{v} \text { tween } \\
20 \text { in distilled } \\
\left.\mathrm{H}_{2} \mathrm{O}\right) .\end{array}$ & $\begin{array}{l}\text { Normal } \\
\text { horse } \\
\text { serum } \\
\text { (NHS 10\% } \\
\text { V/v, Sigma; } \\
\text { H0146) in } \\
\text { distilled } \\
\mathrm{H}_{2} \mathrm{O} \text {. }\end{array}$ & $\begin{array}{l}\text { Rabbit-anti- } \\
\text { GFAP (Abcam; } \\
\text { ab7260) at } \\
1 / 250 \text { in NHS } \\
\text { (5\% v/v, Sigma; } \\
\text { H1270). }\end{array}$ & $\begin{array}{l}\text { Alexa fluor, donkey-anti-rabbit } 568 \\
\text { (0.8mg/mL, Life Technologies; } \\
\text { A10042) in BSA (1\% v/v, Sigma; } \\
\text { A604), foetal bovine serum (FBS } \\
2 \% \text { v/v, Bovogen Biologicals; SFBS- } \\
\text { F) in 1x PBS. }\end{array}$ \\
\hline
\end{tabular}

\subsection{Quantification and statistical analysis}

All data were analysed using GraphPad Prism version 9.0. Normality was assessed using a Shapiro-Wilk test. Following confirmation of normality, statistical significance between groups was identified with a two-way analysis of variance (ANOVA) and appropriate post hoc tests for multiple comparisons or a student's T-test. A Pearson's correlation coefficient was used to identify a statistical association between primary outcome measures which were graphed using a simple linear regression. A $p$ value of $<0.05$ was considered statistically significant. 


\section{Results}

\subsection{5-FU significantly increases TIMP-1 expression at subacute timepoints and IL-6R expression at chronic timepoints}

The expression of key neuroinflammatory genes of interest, including tissue inhibitor of metalloproteinase 1 (TIMP-1; Fig. 1A), matrix metalloproteinase 9 (MMP-9; Fig. 1B), MMP-2 (Fig. 1C), interleukin-6 receptor (IL-6R; Fig. 1D), B-cell lymphoma 2 (BCL2; Fig. 1E) and brain derived neurotrophic factor (BDNF; Fig. 1E) were assessed using RT-PCR. 5-FU treatment caused a significant increase to TIMP-1 expression, which regulates the proteolytic activity of MMPs, at the subacute timepoint when compared to controls (Fig. $1 A ; p=0.031$ ). While there was no significant change to MMP-9 and MMP-2 expression following 5-FU (Fig. 1B \& C respectively), TIMP-1 positively correlated with the combined expression of MMP-2 and MMP-9 $\left(R^{2}=0.192, p=0.037\right.$; data not shown). Suggesting the increase in TIMP-1 expression may be a compensatory mechanism to account for the increased expression of combined MMPs and thus increased proteolytic activity within the CNS.

IL-6 is a pleiotropic cytokine which can have pro- or anti-inflammatory effects depending on the signalling pathway activated through binding to the membrane bound or soluble form of the IL-6R [27]. When bound to the soluble IL6R, IL-6 is capable of activating glycoprotein 130 (gp130) to mediate its pro-inflammatory action [28]. Accordingly, the expression of IL-6R was quantified to investigate the impact of 5-FU on IL-6 signalling. 5-FU treatment caused a significant increase in IL-6R expression at chronic timepoints when compared to controls (Fig. 1D; $p=0.033$ ), highlighting the potential for the involvement of IL-6 signalling in central neurotoxicity.

Although B-cell lymphoma 2 (BCL2) and brain derived neurotrophic factor (BDNF) have established roles in protecting neurogenesis through anti-apoptotic and trophic actions respectively [29,30], neither were found to be altered by 5-FU treatment (Fig. 1E \& F).

\subsection{5-FU markedly reduces neurogenesis at acute timepoints following treatment}

To investigate the possibility of direct neurotoxicity, the impact of 5-FU on neurogenesis was assessed using DCX, a specific marker of newly formed neurons within the dentate gyrus, a key site of hippocampal neurogenesis. 5-FU treatment significantly decreased positive DCX staining within the dentate gyrus at chronic timepoints compared to controls (Fig. $2 A \& B ; p=0.034$ ). Clear clustering of day 1 and day 32 controls was observed, with the means of day 1 and day 32 controls being determined as significantly different by a student's T-test $(p=0.008)$. As such, a sub-analysis was performed between day 1 controls $(\mathrm{N}=3)$ and the percent area of positive DCX stain seen at acute timepoints. This revealed that a significant decrease in DCX staining was present between time matched control and treated animals (Fig. 2C; $\mathrm{p}=0.003$ ), highlighting that 5-FU decreases neurogenesis in the acute setting following treatment. 
Given the role of BCL2 and BDNF in neurogenesis, the relationship between the expression of these two factors and DCX was investigated. A strong positive correlation was observed between BCL2 and DCX expression (Fig. 2D; $\mathrm{R}^{2}=0.422$, $\mathrm{p}=0.0006$ ). However, no correlation between DCX and BDNF expression was established.

\subsection{5-FU induced acute microglial activation in brain regions associated with cognition}

The activation of microglia, which are known as first responders within the CNS and often initiate neuroinflammation when exposed to pathological stimuli [31], was assessed by determining the percent area of positive Iba1 stain (Fig. 3A) in the prefrontal cortex (Fig. 3B), hypothalamus (Fig. 3C) and midbrain (Fig. 3D), as well as the CA3 (Fig. 3E), CA1 (Fig. 3F) and dentate gyrus (Fig. 3G) regions of the hippocampus. Following 5-FU treatment, the expression of Iba1 significantly increased at acute timepoints in the prefrontal cortex (Fig. 3B; $p=0.026$ ), as well as the CA3 region (Fig. 3E; $p=0.028$ ) and dentate gyrus (Fig. 3G; $p=0.005$ ) of the hippocampus when compared to controls, all of which are brain regions critical to cognitive function. Iba1 expression levels then decreased across subacute and chronic timepoints, with levels close to what was seen in controls observed in the chronic setting. This trend of microglial activation was reflected in the hypothalamus (Fig. 3C), midbrain (Fig. 3D) and CA1 region of the hippocampus (Fig. 3F), however results were not significantly different from those of controls.

\subsection{5-FU induced global astrocyte reactivity, with most activation seen at chronic timepoints}

The reactivity of astrocytes, key supportive cells within the CNS that play a role in immune signalling, was assessed using GFAP (Fig. 4A) in the prefrontal cortex (Fig. 4B), hypothalamus (Fig. 4C) and midbrain (Fig. 4D) as well as the CA3 (Fig. 4E), CA1 (Fig. 4F) and dentate gyrus (Fig. 4G) regions of the hippocampus. Following 5-FU, the expression of GFAP significantly increased across multiple brain regions and at varying timepoints post-treatment when compared to controls. Specifically, in the prefrontal cortex, levels of GFAP expression were highest at the subacute timepoint (Fig. 4B; p<0.0001). This was reflected in the CA3 region of the hippocampus (Fig. 4E; $p=0.048$ ). All other regions displayed varying levels of GFAP expression within the acute and subacute setting, with highest levels of expression seen at chronic timepoints when compared to controls. Specifically, these regions included the hypothalamus (Fig. 4C; $p=0.0003$ ) and midbrain (Fig. 4D; $p=0.005$ ) as well as the CA1 region (Fig. 4F; $\mathrm{p}=0.0003$ ) and dentate gyrus (Fig. 4G; $\mathrm{p}<0.0001$ ) of the hippocampus.

\subsection{Astrocyte activation correlates with the neuroinflammatory markers IL-6R, MMP-2 and TIMP1}

The relationship between the activation of glia cells and the expression of neuroinflammatory markers IL6R, MMP-9, MMP-2 and TIMP-1 was investigated following 5-FU treatment. The activation of astrocytes in the hypothalamus, reflected by GFAP expression, shared a positive correlation with the expression of IL6R (Fig. 5A; $\left.R^{2}=0.213, p=0.023\right)$, MMP-2 in the prefrontal cortex (Fig. 5B; $R^{2}=0.373, p=0.003$ ), and TIMP-1 
in both the prefrontal cortex and CA3 region of the hippocampus (Fig. 5C; $R^{2}=272, p=0.013, \&$; $\left.R^{2}=0.174, p=0.042\right)$. There were no significant correlations observed between the expression of GFAP and neuroinflammatory markers in the CA1 region and dentate gyrus of the hippocampus as well as the midbrain (Supplementary Table 1). Furthermore, no significant correlations were observed between microglial activation, reflected by lba1 expression, and neuroinflammatory markers within any brain region investigated (Supplementary Table 2).

\subsection{Astrocyte activation shares a negative correlation with neurogenesis following 5-FU}

The relationship between the activation of glial cells and neurogenesis, reflective of neuroinflammatory and neurodegenerative processes respectively, was investigated by assessing the correlation between the expression of Iba1 and GFAP with both DCX and BCL2 expression following 5-FU. A significant negative correlation was observed between the expression of GFAP and both DCX and BCL2 within the CA1 region (Fig. 6A; $R^{2}=0.199, p=0.029 \& B ; R^{2}=0.225, p=0.019$ ) and the dentate gyrus (Fig. $6 C ; R^{2}=0.201, p=0.028 \&$ $D ; R^{2}=0.223, p=0.020$ ) of the hippocampus, as well as the midbrain (Fig. 6E; $R^{2}=0.166, p=0.048$ \& $R^{2}=0.221, p=0.021$ ) and hippocampus (Fig. 6G; $R^{2}=0.283, p=0.008 \& H ; R^{2}=0.243, p=0.014$ ). No significant correlation was established between the expression of GFAP and both DCX and BCL2 in the prefrontal cortex and CA3 region of the hippocampus (data not shown). Furthermore, no significant correlations were established between the expression of Iba1 and both DCX and BCL2 in any brain regions.

\section{Discussion}

The neuropsychological symptoms related to chemotherapy treatment remain a major, unresolved challenge with prevention hampered by insufficient understanding of their pathophysiology. Long-term neuroimmune changes have been identified as a hallmark feature shared by these symptoms [11], although, the exact timeline of mechanistic events preceding neuroinflammation, and the relationship between different glial cells driving this neuroinflammatory response, remain unclear. Here we provide a longitudinal study of region-specific, neuroimmune changes occurring in response to systemically administered 5-FU, and position impaired neurogenesis along with microglial activation as key initiating events in the resulting neuroinflammatory sequelae, perpetuated by astrocytic reactivity. We have shown for the first time in vivo that, after 5-FU, impaired neurogenesis may be related to reduced BCL2 expression and, following the acute activation of microglia, MMP activity and IL- 6 signalling share a clear relationship with astrocyte reactivity (Fig. 7). These data underscore the acute origins of chronic neuropsychological symptoms highlighting the importance of neuroprotective strategies in acute supportive care.

We have shown that DCX staining was significantly reduced within 24 and 48 hours of systemic administration of 5-FU, establishing that newly born neuronal progenitor cells have been targeted, suggestive of direct cytotoxicity. To confirm whether there is active cell death occurring, additional 
markers of apoptosis and autophagy could be investigated in future studies. This supports the hypothesis that the inhibition of adult hippocampal neurogenesis by chemotherapy drives the development of the neuropsychological symptoms associated with these treatments [32]. Furthermore, activation of microglia, but not astrocytes, was seen at acute timepoints following 5-FU. Our observation indicates that chemotherapy agents act directly on microglia, driving and initiating a neuroinflammatory response consistent with the findings of a sophisticated in vitro experiment that demonstrated significant reactivity of microglial, but not astrocyte, cell cultures when exposed to the chemotherapy agent methotrexate [33]. Instead, the treatment of astrocytes with conditioned media from methotrexateexposed microglia initiated broad astrocytic reactivity, establishing that microglia may respond directly to chemotherapeutics, but astrocytes require reactive microglia to drive their activation [33]. In the current study, increased lba1 staining was observed at acute timepoints, and a global increase to GFAP staining was seen at subacute and chronic timepoints and is to the best of our knowledge, the first to confirm the in vivo temporal relationship between glial cell activation, following chemotherapy treatment. These findings, along with the observed decrease in levels of DCX following 5-FU, support the possibility of 5-FU acting directly within the CNS to initiate the neuroimmune changes seen at acute timepoints following treatment.

The presence of cytotoxic injury implies 5-FU has the capacity to access the brain to induce damage. Unlike many other chemotherapy drugs, 5 -FU has a small molecular weight and therefore is capable of crossing the BBB [34]. However, despite 5-FU being routinely utilised for the treatment of CNS malignancies, its efficacy is somewhat limited by the agent's high polarity [35]. As such, the concentration of 5-FU that truly accesses the brain and the capacity for this dose to induce neurotoxicity remains unclear. A further consideration in the ability of 5-FU to induce direct cytotoxicity is its short half-life of only 8 to 20 minutes in vivo, and its rapid catabolism in the liver [36]. As such, identifying the presence and distribution of 5-FU within the brain, using emerging techniques such as spatial metabolomics with mass spectrometry imaging, which provides greater sensitivity to low yield, small molecules compared to traditional techniques [37], is critical to understanding the role of direct cytotoxicity in the neuroimmune changes occurring post 5-FU treatment, as well as its distribution among critical brain regions such as the hippocampus.

While it is plausible that 5-FU enters the CNS to directly damage newly born neuronal progenitor cells and activate microglia, other triggers cannot be ignored, particularly given that we used only a single dose of 5 -FU. Inflammatory processes are documented to induce neurodegeneration and cell death, with neuroinflammation being a hallmark feature shared by numerous neurodegenerative disorders [38]. Importantly, intense peripheral inflammation and the generation of associated danger signals (e.g. DAMP's, PAMP's and MAMP's) are commonly induced by a range of chemotherapy agents. These inflammatory responses are triggered by cytotoxic injury of chemotherapy drugs against tumor cells and the collateral damage they cause to healthy tissue, in particular the highly proliferative intestinal mucosa [18]. For example, gastrointestinal mucositis and breakdown of the mucosal barrier, permits translocation of gut-derived inflammatory mediators and bacterial endotoxins such as lipopolysaccharide (LPS), into systemic circulation where they are able to impact BBB integrity [39]. Apoptosis within the intestinal 
mucosa and resulting peaks in peripheral endotoxin levels have been observed at 6 and 24 hours following chemotherapy treatment respectively in mice. In that same study, damage to BBB integrity was identified at 24, 48 and 72 hours post treatment, highlighting a clear time course of events facilitating peripheral to central communication [19]. With increases to BBB permeability and the movement of peripheral inflammatory mediators into the CNS, it is possible that these peripherally-derived mediators act to induce the acute damage to neuronal progenitor cells and activation of microglia seen following 5FU treatment, or may work in conjunction with 5-FU to induce neuronal cell death [39-41].

After the transient increase to microglial activation observed at acute timepoints following 5-FU, the downstream neuroinflammatory response characterised in our study involved increases to TIMP-1 and associated MMP expression as well as IL-6R, with long term astrocyte reactivity. A compensatory increase to TIMP-1 expression, reflective of increases to the overall levels of MMPs present within the CNS, is seen in astrocytes when exposed to conditioned media from activated microglial cell cultures [42]. We identified a positive correlation between TIMP-1 expression and astrocyte activation within the prefrontal cortex and CA3 region of the hippocampus, with peaks in expression and activation observed at subacute timepoints. Given that microglia are known to produce MMPs [43], it is likely that the acute activation of microglia induces increased TIMP-1 expression, to compensate for increased MMP activity. In the chronic setting following 5-FU treatment, TIMP-1 expression returned to levels similar to those seen at baseline. However, IL-6R expression peaked in the chronic setting and this was positively correlated with the level of astrocyte activation observed in the hypothalamus. Trans signalling via the IL-6R is critical in mediating the pro-inflammatory action of IL-6 [28] and as such IL-6 signalling may underlie the chronic activation of astrocytes and resulting neuroinflammation following 5-FU. Therefore, our data imply that acute microglial activation and resulting MMP activity are initiating factors in downstream astrocyte activation and TIMP-1 expression within the subacute setting and IL-6 signalling as a critical mediator perpetuating chronic astrocyte reactivity following 5 -FU.

Our evidence suggests that chronic neuropsychological symptoms have acute cytotoxic origins. This new knowledge challenges the current management guidelines which are fundamentally reactive. In addition to being variably implemented, neuropsychological symptoms are largely rehabilitated through group training, which entails repetitive completion of tasks posing a mental challenge, as well as psychological intervention and pharmacotherapy when symptoms develop [44]. If our data are indeed translatable to humans, we suggest that ongoing work should be focussed on protecting the brain in the acute phases of chemotherapy treatment by either reinforcing the BBB or mitigating peripheral signals that drive neurological changes.

Our study is the first to longitudinally characterise the neuroimmune changes occurring post 5-FU treatment, however it has some limitations. Notably, we did not undertake behavioural phenotyping in these animals as the main aim of this study was to investigate the timeline of molecular events preceding the chronic neuroinflammation seen following chemotherapy. While this neuroinflammatory response is proposed to underlie the neuropsychological side effects of chemotherapy treatment, the inclusion of behavioural analyses probing cognitive and psychological function would have introduced 
significant confounding factors without providing additional data relevant to our fundamental research question. As established by Mandillo et al. stress related to the repetitive handling required by a battery of behavioural assessments, is the primary confounding variable affecting reliability and reproducibility of these tests $[45,46]$. Given that stress can drive the development of neuronal damage, including both neuroinflammation and deficits in neurogenesis [23,47], the inclusion of behavioural analyses and the resulting stress introduced by repeated handling may have masked the true effects of 5-FU on the outcome measures reported in this study. A further limitation is that all transcriptomic analyses were performed using the frontal cortex of the brain and as such no conclusions can be made regarding regional changes in the expression of the genes investigated. These analyses could, however, provide further clarification to the physiological changes occurring in critical brain regions, such as the hippocampus, following chemotherapy and thus is an avenue for future research to build on the time course of neurological events defined by the current study.

\section{Conclusions And Future Directions}

The neuropsychological deficits associated with chemotherapy treatment remain a priority concern in the field of supportive oncology. This study has, for the first time, described the longitudinal timeline of neuroimmune events present following 5-FU treatment and identified impaired neurogenesis and acute activation of microglia as probably initiating factors underlying downstream neuroinflammation, sustained by astrocyte reactivity. These findings highlight the temporal dynamics of neurotoxicity and neuroinflammation and that significant and persistent neuroimmune changes can occur within the brain following a single dose of systemically administered chemotherapy. This strongly suggests that protecting the brain within the acute phases of treatment may be critical to preventing neuropsychological symptom development and warrants further research to identify modifiable triggers of acute neurotoxicity. We suggest that this preclinical model is well suited to enable continued investigations of this mechanism and test new neuroprotective interventions.

\section{Declarations}

Funding: This work was supported by a Research Training Program Australia Stipend, The Doctor Chun Chung Wong and Madam So Sau Lam Memorial Postgraduate Cancer Research Top-Up Scholarship (CBS) and an NHMRC CJ Martin Biomedical Research Fellowship (HRW).

Competing Interests: Joanne Bowen has received research funding from AstraZeneca, Helsinn Healthcare, Pfizer Pharmaceuticals, PanTheryx and Puma Biotechnology Inc. Hannah Wardill has received research funding from Nutricia (Danone) Research. Remaining authors have no conflicts of interest to declare.

Acknowledgements: Ines Semendric for providing technical assistance. Adelaide Microscopy, University of Adelaide and Bioresources Staff, SAHMRI. 
Author Contributions: Courtney Subramaniam:Methodology, Investigation, Formal Analysis, Writing Original Draft; Hannah Wardill: Supervision, Conceptualisation, Formal Analysis, Writing - Review \& Editing; Maya Davies: investigation; Vivien Heng: Investigation; Marc Gladman: Supervision, Writing Review \& Editing; Joanne Bowen: Supervision, Conceptualisation, Writing - Review \& Editing.

Data Availability: The datasets generated within this study are not currently available to the public. However, can be made available upon request to the corresponding author.

Ethics Approval: The study was approved by the Animal Ethics Committee of the South Australian Health and Medical Research Institute (SAHMRI, \#SAM20-034) and complied with the National Health and Research Council (Australia) Code of Practice for Animal Care in Research and Training (2014).

\section{Consent To Participate: N/A.}

\section{Consent To Publish: N/A.}

\section{References}

1. Nurgali K, Jagoe RT, Abalo R (2018) Editorial: Adverse Effects of Cancer Chemotherapy: Anything New to Improve Tolerance and Reduce Sequelae? Front Pharmacol 9:245

2. Apple AC et al (2017) Subtle hippocampal deformities in breast cancer survivors with reduced episodic memory and self-reported cognitive concerns. Neuroimage Clin 14:685-691

3. Apple AC et al (2018) Hippocampal functional connectivity is related to self-reported cognitive concerns in breast cancer patients undergoing adjuvant therapy. Neuroimage Clin 20:110-118

4. Kesler SR, Kent JS, O'Hara R (2011) Prefrontal cortex and executive function impairments in primary breast cancer. Arch Neurol 68(11):1447-1453

5. Yang $Y$ et al (2017) The relationship between cancer patient's fear of recurrence and chemotherapy: A systematic review and meta-analysis. J Psychosom Res 98:55-63

6. Souza $R$ et al (2020) Factors associated with sleep quality during chemotherapy: An integrative review. Nurs Open 7(5):1274-1284

7. Selamat MH et al (2014) Chemobrain experienced by breast cancer survivors: a meta-ethnography study investigating research and care implications. PLoS ONE 9(9):e108002

8. Ramsey I et al (2021) A core set of patient-reported outcomes for population-based cancer survivorship research: a consensus study. J Cancer Surviv 15(2):201-212

9. Huang J et al (2016) Symptom Clusters in Ovarian Cancer Patients With Chemotherapy After Surgery: A Longitudinal Survey. Cancer Nurs 39(2):106-116

10. Sullivan CW et al (2018) Stability of Symptom Clusters in Patients With Breast Cancer Receiving Chemotherapy. J Pain Symptom Manage 55(1):39-55

11. Santos JC, Pyter LM (2018) Neuroimmunology of Behavioral Comorbidities Associated With Cancer and Cancer Treatments. Front Immunol 9:1195 
12. Cherry JD, Olschowka JA, O'Banion MK (2014) Neuroinflammation and M2 microglia: the good, the bad, and the inflamed. J Neuroinflammation 11:98

13. Michopoulos V et al (2017) Inflammation in Fear- and Anxiety-Based Disorders: PTSD, GAD, and Beyond. Neuropsychopharmacology 42(1):254-270

14. Troubat $R$ et al (2021) Neuroinflammation and depression: A review. Eur J Neurosci 53(1):151-171

15. Stephenson J et al (2018) Inflammation in CNS neurodegenerative diseases. Immunology 154(2):204-219

16. George RP et al (2021) Neuroimmune reactivity marker expression in rodent models of chemotherapy-induced cognitive impairment: A systematic scoping review. Brain Behav Immun 94:392-409

17. Subramaniam CB et al (2020) The microbiota-gut-brain axis: An emerging therapeutic target in chemotherapy-induced cognitive impairment. Neurosci Biobehav Rev 116:470-479

18. Bowen $\mathrm{J}$ et al (2019) The pathogenesis of mucositis: updated perspectives and emerging targets. Support Care Cancer 27(10):4023-4033

19. Wardill HR et al (2016) Irinotecan-Induced Gastrointestinal Dysfunction and Pain Are Mediated by Common TLR4-Dependent Mechanisms. Mol Cancer Ther 15(6):1376-1386

20. Sritawan $\mathrm{N}$ et al (2020) Metformin alleviates memory and hippocampal neurogenesis decline induced by methotrexate chemotherapy in a rat model. Biomed Pharmacother 131:110651

21. Egeland $\mathrm{M}$ et al (2017) Depletion of adult neurogenesis using the chemotherapy drug temozolomide in mice induces behavioural and biological changes relevant to depression. Transl Psychiatry 7(4):e1101

22. Jiang ZG et al (2018) PAN-811 prevents chemotherapy-induced cognitive impairment and preserves neurogenesis in the hippocampus of adult rats. PLoS ONE 13(1):e0191866

23. Dioli $\mathrm{C}$ et al (2019) Chronic stress triggers divergent dendritic alterations in immature neurons of the adult hippocampus, depending on their ultimate terminal fields. Transl Psychiatry 9(1):143

24. Chesnokova V, Pechnick RN, Wawrowsky K (2016) Chronic peripheral inflammation, hippocampal neurogenesis, and behavior. Brain Behav Immun 58:1-8

25. Roxburgh CS, McMillan DC (2014) Cancer and systemic inflammation: treat the tumour and treat the host. Br J Cancer 110(6):1409-1412

26. Livak KJ, Schmittgen TD (2001) Analysis of relative gene expression data using real-time quantitative PCR and the 2(-Delta Delta C(T)) Method. Methods 25(4):402-408

27. Erta M, Quintana A, Hidalgo J (2012) Interleukin-6, a major cytokine in the central nervous system. Int J Biol Sci 8(9):1254-1266

28. Rose-John S (2012) IL-6 trans-signaling via the soluble IL-6 receptor: importance for the proinflammatory activities of IL-6. Int J Biol Sci 8(9):1237-1247

29. Zhang R et al (2006) Bcl-2 enhances neurogenesis and inhibits apoptosis of newborn neurons in adult rat brain following a transient middle cerebral artery occlusion. Neurobiol Dis 24(2):345-356 
30. Numakawa T, Odaka H, Adachi N (2017) Actions of Brain-Derived Neurotrophic Factor and Glucocorticoid Stress in Neurogenesis. Int J Mol Sci, 18(11)

31. Cătălin B et al (2013) Microglia: first responders in the central nervous system. Rom J Morphol Embryol 54(3):467-472

32. Pereira Dias G et al (2014) Consequences of cancer treatments on adult hippocampal neurogenesis: implications for cognitive function and depressive symptoms. Neuro Oncol 16(4):476-492

33. Gibson EM et al (2019) Methotrexate Chemotherapy Induces Persistent Tri-glial Dysregulation that Underlies Chemotherapy-Related Cognitive Impairment. Cell 176(1-2):43-55e13

34. Formica V et al (2006) 5-Fluorouracil can cross brain-blood barrier and cause encephalopathy: should we expect the same from capecitabine? A case report on capecitabine-induced central neurotoxicity progressing to coma. Cancer Chemother Pharmacol, 58(2): p. 276-8

35. Shinde $\mathrm{G}$ et al (2020) Enhanced brain targeting efficiency using 5-FU (fluorouracil) lipid-drug conjugated nanoparticles in brain cancer therapy. Prog Biomater 9(4):259-275

36. Diasio RB, Harris BE (1989) Clinical pharmacology of 5-fluorouracil. Clin Pharmacokinet 16(4):215237

37. Alexandrov T (2020) Spatial Metabolomics and Imaging Mass Spectrometry in the Age of Artificial Intelligence. Annu Rev Biomed Data Sci 3:61-87

38. Guzman-Martinez L et al (2019) Neuroinflammation as a Common Feature of Neurodegenerative Disorders. Front Pharmacol 10:1008

39. Wardill HR et al (2016) Cytokine-mediated blood brain barrier disruption as a conduit for cancer/chemotherapy-associated neurotoxicity and cognitive dysfunction. Int $\mathrm{J}$ Cancer 139(12):2635-2645

40. Perez-Dominguez M et al (2019) The detrimental effects of lipopolysaccharide-induced neuroinflammation on adult hippocampal neurogenesis depend on the duration of the proinflammatory response. Neural Regen Res 14(5):817-825

41. Zhao J et al (2019) Neuroinflammation induced by lipopolysaccharide causes cognitive impairment in mice. Sci Rep 9(1):5790

42. Welser-Alves JV, Crocker SJ, Milner R (2011) A dual role for microglia in promoting tissue inhibitor of metalloproteinase (TIMP) expression in glial cells in response to neuroinflammatory stimuli. $J$ Neuroinflammation 8:61

43. Könnecke H, Bechmann I (2013) The role of microglia and matrix metalloproteinases involvement in neuroinflammation and gliomas. Clin Dev Immunol, 2013: p. 914104

44. Runowicz CD et al (2016) American Cancer Society/American Society of Clinical Oncology Breast Cancer Survivorship Care Guideline. CA Cancer J Clin 66(1):43-73

45. Mandillo $S$ et al (2008) Reliability, robustness, and reproducibility in mouse behavioral phenotyping: a cross-laboratory study. Physiol Genomics 34(3):243-255 
46. Saré RM, Lemons A, Smith CB (2021) Behavior Testing in Rodents: Highlighting Potential Confounds Affecting Variability and Reproducibility.Brain Sci, 11(4)

47. Calcia MA et al (2016) Stress and neuroinflammation: a systematic review of the effects of stress on microglia and the implications for mental illness. Psychopharmacology 233(9):1637-1650

Figures

A

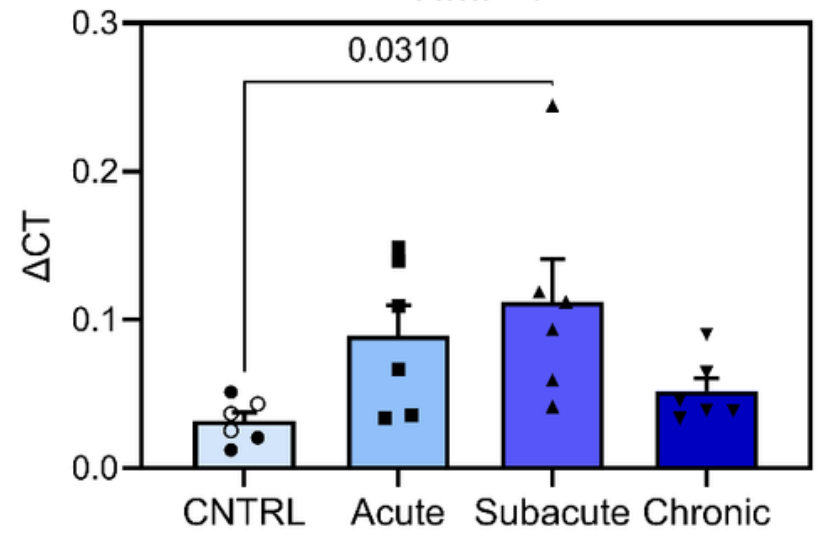

C

0.5
0.4
$5^{0.3}$
MMP-2

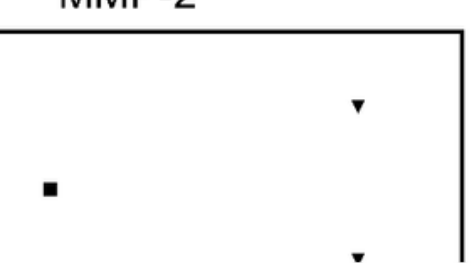

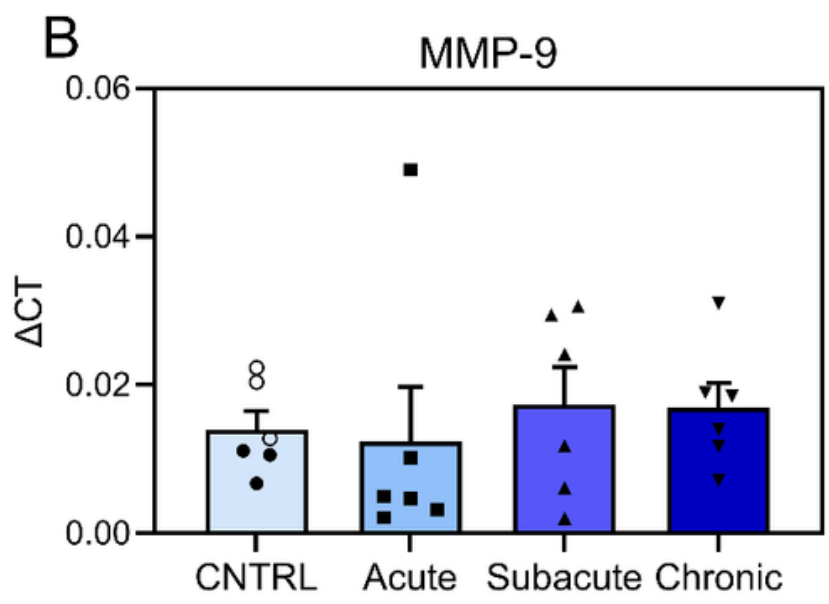

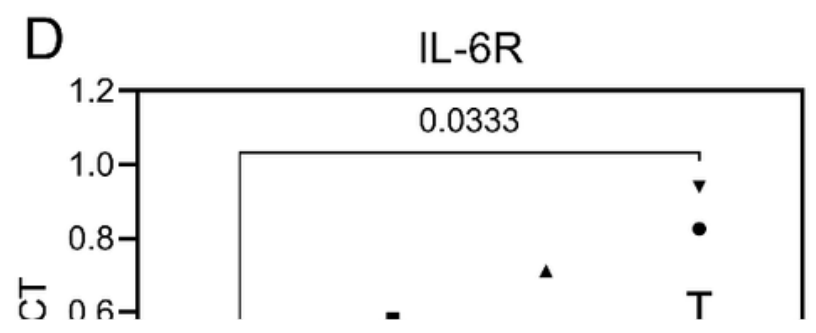




\section{Figure 1}

Expression of (A) TIMP-1, (B) MMP-9, (C) MMP-2, (D) IL-6R, (E) BCL2 and (F) BDNF, quantified using the $\triangle \mathrm{CT}$ method, at acute, subacute and chronic timepoints following 5-FU. All data is presented as mean \pm SEM, represent day 1 controls; 0 represent day 32 controls.

\section{Figure 2}

Representative images from the dentate gyrus of control and treated animals at chronic timepoints show that 5-FU decreased DCX expression (red; nuclei, DAPI blue, scale bar 50 $\mu \mathrm{m}$ ) (A). Quantification of the percent area stain is presented for DCX in the dentate gyrus (B). Sub-analysis of day 1 controls and acute timepoints show a significant decrease in DCX expression (C). DCX expression shares a positive correlation with BCL2 (D) but not BDNF (E) expression following 5-FU. All data is presented as mean \pm $\mathrm{SEM}, \bigcirc$ represent day 1 controls; $\bigcirc$ represent day 32 controls.

\section{Figure 3}

Representative images from the apex of the dentate gyrus of control and treated animals at the acute

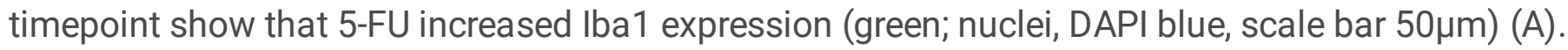
Quantification of the percent area stain is presented for the prefrontal cortex (B), hypothalamus (C) and midbrain (D) as well as the CA3 (E), CA1 (F) and dentate gyrus (G) regions of the hippocampus. All data is presented as mean $\pm \mathrm{SEM}$, represent day 1 controls; $\bigcirc$ represent day 32 controls.

\section{Figure 4}

Representative images from control and treated animals at the chronic timepoint show that 5-FU increased GFAP expression (red; nuclei, DAPI blue, scale bar $50 \mu \mathrm{m}$ ) (A). Quantification of the percent area stain is presented for the prefrontal cortex (B), hypothalamus (C) and midbrain (D) as well as the CA3 (E), CA1 $(F)$ and dentate gyrus $(\mathrm{G})$ regions of the hippocampus. All data is presented as mean \pm SEM represent day 1 controls; 9 represent day 32 controls. 

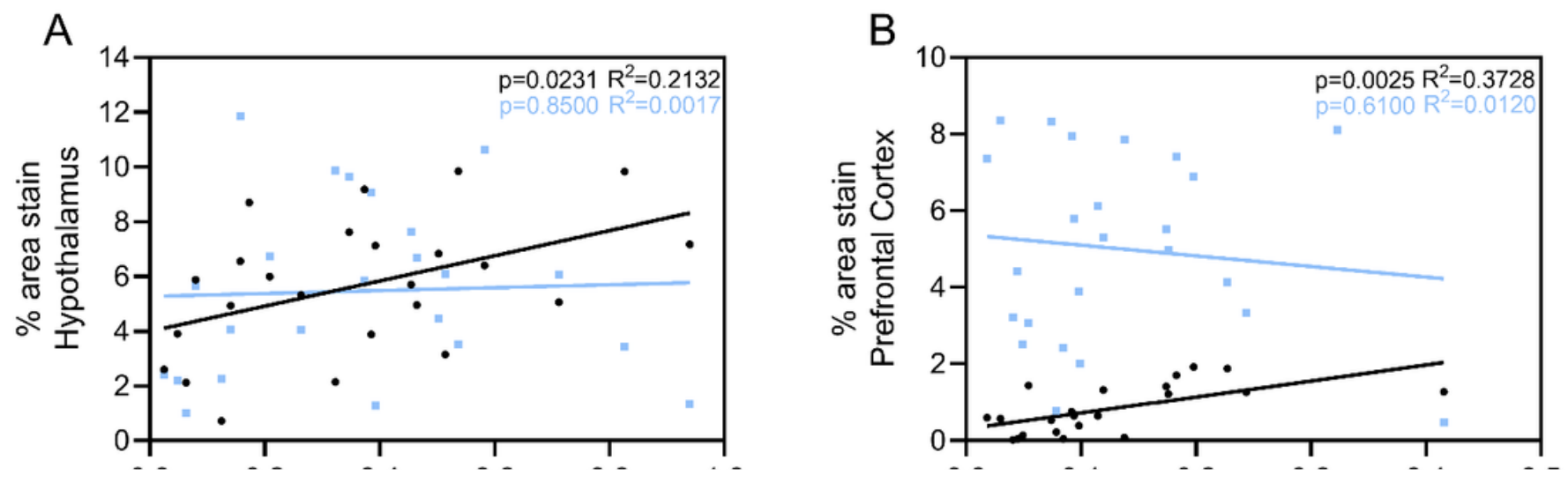

Figure 5

Pearson's correlation established that the expression of GFAP, but not Iba1, positively correlates with IL-6R expression in the hypothalamus (A), MMP-2 expression in the prefrontal cortex (B) and TIMP-1 expression in both the prefrontal cortex (C) and CA3 region of the hippocampus (D).

\section{Figure 6}

Pearson's correlation established that the expression of DCX and BCL2 negatively correlates with the expression of GFAP, but not Iba1, in the CA1 region $(A, B)$ and dentate gyrus $(C, D)$ of the hippocampus as well as the midbrain $(E, F)$ and hypothalamus $(G, H)$. 


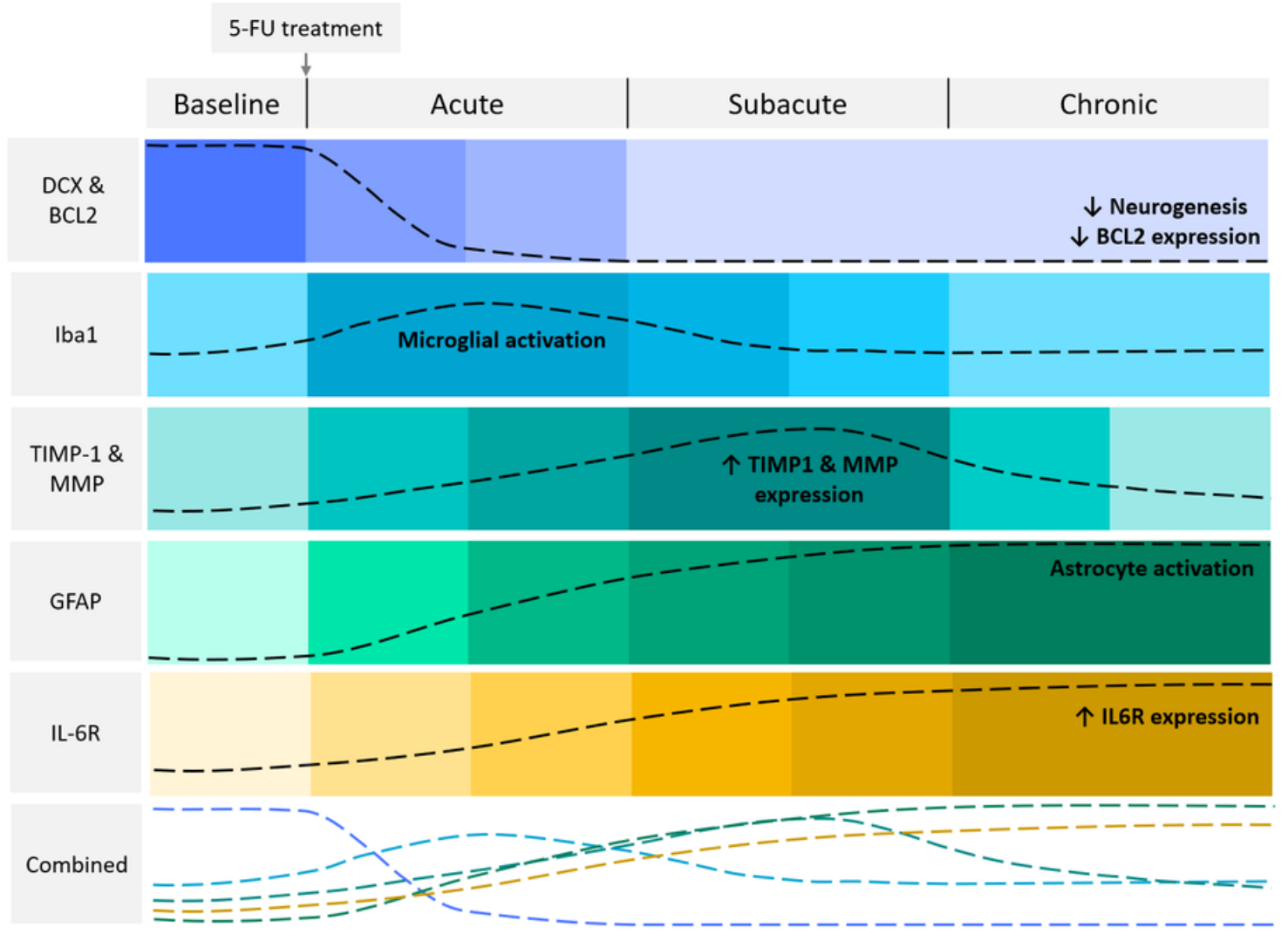

\section{Figure 7}

Diagrammatic representation of the neuroimmune changes observed following 5-FU treatment. From baseline expression levels, DCX and associated BCL2 expression decreased at acute timepoints and maintained this level of expression over subacute and chronic timepoints. Microglial activation increased at acute timepoints but returned to what was seen at baseline in the chronic setting. TIMP-1 and associated MMP expression was highest at subacute timepoints. Astrocyte activation and associated IL$6 \mathrm{R}$ expression, were highest in the chronic setting.

\section{Supplementary Files}

This is a list of supplementary files associated with this preprint. Click to download.

- SupplementaryFigure1.docx 